\title{
HIERARCHICAL MODELING OF THE HEAT EQUATION IN A HETEROGENEOUS PLATE
}

\author{
ANA CAROLINA CARIUS
}

ALEXANDRE L. MADUREIRA

\begin{abstract}
In this work we investigate the modeling of heterogeneous plates, where the length scale of the heterogeneity can be much smaller than the area of the plate's middle surface. We derive a two-dimensional model for the original problem, and the resulting PDEs not only have rough coefficients but also depend on the thickness, resulting in a singularly perturbed problem. We employ asymptotic techniques to show that, as the plate thickness tends to zero, our model converges to the exact solution. To tame the numerical troubles of the resulting model we use finite elements methods of multiscale type.
\end{abstract}

\section{INTRODUCTION}

The challenge of solving PDEs in beams, plates and shells has historically attracted researchers from different fields, not only because of the importance of the physical problems demanding such task, but also because of the beautiful problems arising from the endeavor. Focusing on plates, the first necessary step is to perform some sort of dimension reduction, i.e., model a three-dimensional problem with a two-dimensional model. Hopefully, the resulting equations are easier to solve, and the final solution approximates in some sense the exact solution of the original problem.

There are basically three known ways, not always exclusive, to obtain plate models. Probably the most common arguments are based on physical properties of the underlying problem, often combined with some mathematical reasoning. It is also possible to derive the models using asymptotic techniques, usually with a sound mathematical basis, and the results easier

Date: December 10, 2007.

Key words and phrases. Homogenization, Dimension reduction, Residual Free Bubbles, Multiscale Finite Element Method.

The authors would like to thank Gabriel Barrenechea and Frédéric Valentin for the kind invitation to participate in the mini-symposium Stabilized and Multiscale Finite Element Methods, at the Second Chilean Workshop on Numerical Analysis of Partial Differential Equations - WONAPDE 2007. Gabriel and Fred were important influences in the making of this present work.

The first author had support from CAPES, and the second author was partially supported by the CNPq/Brazil Projects 306104/2004-0 and 486026/2006-0. 
to justify a posteriori. The asymptotic arguments consist in taking the plate thickness to zero and finding "limit problems." For instance, linearly elastic plates have as limit biharmonic equations [12]. For heterogeneous materials however, an extra issue arises. There are situations when the attempts of homogenizing the material may lead to different models, depending on which limit is considered first, i.e., homogenization first and then dimension reduction, or the other way around $[10,13]$. This happens even when stationary heat problems are considered [9].

To avoid such undesirable peculiarity, we shall use hierarchical modeling. In such approach, the solution can defined for instance as the minimizer of the potential energy in the subspace of functions that are polynomials in the transverse direction. The higher the polynomial order, the better is the model. Likewise, the thinner is the plate, the better is the approximation $[2,21]$.

In this work we consider the heat equation in a heterogeneous plate of thickness $2 \delta$ given by $P^{\delta}=\Omega \times(-\delta, \delta)$, where $\Omega \subset \mathbb{R}^{2}$ is a bounded open domain with Lipschitz boundary $\partial \Omega$. Let $\partial P_{L}^{\delta}=\partial \Omega \times(-\delta, \delta)$ be the lateral side of the plate, and $\partial P_{ \pm}^{\delta}=\Omega \times\{-\delta, \delta\}$ its

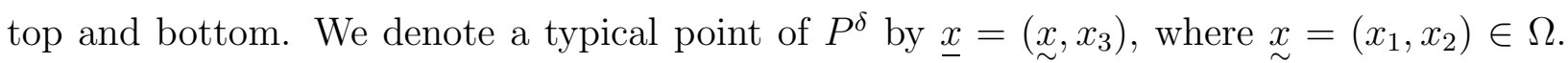
Accordingly, we write $\underline{\nabla}=\left(\underset{\sim}{\nabla}, \partial_{3}\right)=\left(\partial_{1}, \partial_{2}, \partial_{3}\right)$, where $\partial_{i}$ indicates the partial derivative with respect to $x_{i}$. Also, $\partial_{i j}=\partial_{i} \partial_{j}$.

Let $u^{\delta} \in H^{1}\left(P^{\delta}\right)$ be the weak solution of

$$
\begin{gathered}
-\operatorname{div}\left(\stackrel{\left.\underline{A} \underline{\nabla} u^{\delta}\right)=f^{\delta} \quad \text { in } P^{\delta},}{ }\right. \\
u^{\delta}=0 \quad \text { in } \partial P_{L}^{\delta}, \quad a_{33} \frac{\partial u^{\delta}}{\partial n}=g^{\delta} \quad \text { in } \partial P_{ \pm}^{\delta},
\end{gathered}
$$

where $f^{\delta}: P^{\delta} \rightarrow \mathbb{R}$ and $g^{\delta}: \partial P_{ \pm}^{\delta} \rightarrow \mathbb{R}$. The matrix $\underset{=}{A}: P^{\delta} \rightarrow \mathbb{R}_{\mathrm{SYM}}^{3 \times 3}$ is such that

$$
\underline{A}(\underline{x})=\left(\begin{array}{cc}
\underset{\approx}{a} \underset{\sim}{x}) & 0 \\
0 & a_{33}(\underset{\sim}{x})
\end{array}\right),
$$

where $\underset{\approx}{a}: \Omega \rightarrow \mathbb{R}_{\mathrm{SYM}}^{2 \times 2}$, and $a_{33}: \Omega \rightarrow \mathbb{R}$. We also assume that $a_{i j}, f^{\delta}$, and $g^{\delta}$ are $C^{\infty}$ functions, and that there exist constants $\alpha$ and $\beta$ such that

$$
\alpha\|\underline{\xi}\|^{2} \leq \underline{\xi} \cdot \underline{A}(\underline{x}) \underline{\xi}, \quad \underline{\xi} \cdot \underline{A}(\underline{x}) \underline{\eta} \leq \beta\|\underline{\xi}\|\|\underline{\eta}\|,
$$

for all $\underline{\xi}, \underline{\eta} \in \mathbb{R}^{3}$, and for all $\underline{x} \in P^{\delta}$. The norm $\|\cdot\|$ is the Euclidian norm in $\mathbb{R}^{3}$. Note that the heterogeneity is in the horizontal direction. This model mimics a plate with transverse inclusions.

We next describe the contents of this paper. In Section 2, we derive dimensional reduced Partial Differential Equations (PDEs) for the Poisson problem in a heterogeneous plate using 
hierarchical modeling. The resulting PDEs depend on two small parameters, the thickness and the length scale of the heterogeneity, and pose nontrivial numerical challenges [2-4]. Next, in Section 3, we show that our model is asymptotically consistent, i.e., it converges in a proper sense to the solution of the original problem as the plate thickness goes to zero. Modeling error estimates come by after a somewhat lengthy asymptotic analysis of both the exact and approximate solution. A comparison between the related asymptotic expansions yield error estimates in several norms [2], but we restrict ourselves to a $H^{1}$ estimate in a properly scaled plate.

Our model is given by an uncoupled system of two equations, corresponding to the even and odd parts of the solution (with respect to the middle surface). The first part is a diffusion equation with rapidly varying coefficients that does not depend on the plate thickness. The second equation is of reaction-diffusion type with oscillatory coefficients on its diffusive part, and the predominant "reaction" part depends on the thickness. Thus both equations pose formidable numerical troubles, and to tame them we employ finite elements methods of multiscale type. We describe these methods in Section 4, and perform computational experiments using the Residual Free Bubbles (RFB) Method and the Multiscale Finite Element Method in Section 5. In the Appendix, we outline the derivation of the model.

\section{Derivation of the MODEL}

To derive our model for (1), we first characterize the exact solution $u^{\delta}$ in an alternative way. Let

$$
V\left(P^{\delta}\right)=\left\{v \in H^{1}\left(P^{\delta}\right):\left.v\right|_{\partial P_{L}^{\delta}}=0\right\} .
$$

Then $u^{\delta}$ minimizes the potential energy in $V\left(P^{\delta}\right)$, i.e.,

$$
u^{\delta}=\underset{v \in V\left(P^{\delta}\right)}{\arg \min } \mathcal{I}(v), \quad \text { where } \mathcal{I}(v)=\frac{1}{2} \int_{P^{\delta}} \underline{\nabla} v \cdot \underline{A} \underline{\nabla} v d \underline{x}-\int_{P^{\delta}} f^{\delta} v d \underline{x}+\int_{\partial P_{ \pm}^{\delta}} g^{\delta} v d \underline{x} .
$$

Our model solution $\tilde{u}^{\delta}$, which approximates $u^{\delta}$, is defined as the minimizer of the potential energy in the space of functions in of $V\left(P^{\delta}\right)$ which are linear in the transverse direction, i.e.,

$$
\tilde{u}^{\delta}=\underset{v \in V_{1}\left(P^{\delta}\right)}{\arg \min } \mathcal{I}(v)
$$

and $\left.V_{1}\left(P^{\delta}\right)=\left\{v \in V\left(P^{\delta}\right): v\left(\underset{\sim}{x}, x_{3}\right)=v_{0}(\underset{\sim}{x})+x_{3} v_{1} \underset{\sim}{x}\right), v_{0}, v_{1} \in H_{0}^{1}(\Omega)\right\}$.

If we write

$$
\tilde{u}^{\delta}\left(\underset{\sim}{x}, x_{3}\right)=w_{0}(\underset{\sim}{x})+x_{3} w_{1}(\underset{\sim}{x}),
$$


then $w_{0}, w_{1} \in H_{0}^{1}(\Omega)$ are weak solutions for

$$
\left.\left.\left.-2 \delta \operatorname{div}[\underset{\sim}{a} \underset{\sim}{x}) \underset{\sim}{\nabla} w_{0}\right]=\int_{-\delta}^{\delta} f^{\delta}\left(\underset{\sim}{x}, x_{3}\right) d x_{3}+g^{\delta} \underset{\sim}{x}, \delta\right)+g^{\delta} \underset{\sim}{x},-\delta\right) \quad \text { in } \Omega,
$$

$$
\left.-\frac{2 \delta^{3}}{3} \operatorname{div}[\underset{\sim}{a} \underset{\sim}{x}) \underset{\sim}{\nabla} w_{1}\right]+2 \delta a_{33}(\underset{\sim}{x}) w_{1}=\int_{-\delta}^{\delta} f^{\delta}\left(\underset{\sim}{x}, x_{3}\right) x_{3} d x_{3}+\delta\left[g^{\delta}(\underset{\sim}{x}, \delta)-g^{\delta}(\underset{\sim}{x},-\delta)\right] \quad \text { in } \Omega .
$$

Although the above system is simple to obtain, it apparently never appeared in the literature, so we outline its derivation in the Appendix.

\section{Modeling ERror estimate}

In this section we estimate the modeling error with respect to the plate thickness $\delta$. Note that this is a nontrivial question since the domain itself depends on such parameter. Thus we scale the domain to remove such dependence, and compare the solutions in a plate with fixed thickness [12]. Let

$$
P=\Omega \times(-1,1), \quad \partial P_{L}=\partial \Omega \times(-1,1), \quad \partial P_{ \pm}=\Omega \times\{-1,1\} .
$$

Making the change of coordinates $\underline{\hat{x}}=\left(\underset{\sim}{x}, \delta^{-1} x_{3}\right)$, and defining

$$
u(\delta)(\underline{\hat{x}})=u^{\delta}(\underline{x}), \quad f(\underline{\hat{x}})=f^{\delta}(\underline{x}), \quad g(\underline{\hat{x}})=\delta^{-1} g^{\delta}(\underline{x}),
$$

it follows that

$$
\begin{gathered}
\operatorname{div}[\underset{\sim}{a} \underset{\sim}{\hat{\sim}}) \underset{\sim}{\nabla} u(\delta)]+\delta^{-2} \partial_{3}\left[a_{33}(\underset{\sim}{\hat{x}}) \partial_{3} u(\delta)\right]=-f \quad \text { in } P, \\
u(\delta)=0 \quad \text { on } \partial P_{L}, \quad \delta^{-1} a_{33}(\underset{\sim}{\hat{x}}) \partial_{3} u(\delta)=\delta \hat{x}_{3} g \quad \text { on } \partial P_{ \pm} .
\end{gathered}
$$

Assuming that $f$ and $g$ are $\delta$-independent, and considering the asymptotic expansion

$$
u(\delta) \sim u_{0}+\delta^{2} u_{2}+\delta^{4} u_{4}+\cdots,
$$

we formally gather that

$$
\begin{gathered}
\left.\delta^{-2} \partial_{3}\left[a_{33}(\underset{\sim}{\hat{x}}) \partial_{3} u_{0}\right]+\operatorname{div}[\underset{\sim}{a} \underset{\sim}{(\hat{x}}) \underset{\sim}{\nabla} u_{0}\right]+\partial_{3}\left[a_{33}(\underset{\sim}{\hat{x}}) \partial_{3} u_{2}\right]+\cdots=-f \quad \text { in } P, \\
\delta^{-1} a_{33}(\underset{\sim}{\hat{x}}) \partial_{3} u_{0}+\delta a_{33}(\underset{\sim}{\hat{x}}) \partial_{3} u_{2}+\cdots=\delta \hat{x}_{3} g \quad \text { on } \partial P_{ \pm} .
\end{gathered}
$$

Equating terms with same power of $\delta$, we have that on $P$,

$$
\begin{gathered}
\partial_{3}\left[a_{33}(\underset{\sim}{\hat{x}}) \partial_{3} u_{0}\right]=0, \\
\partial_{3}\left[a_{33}(\underset{\sim}{\hat{x}}) \partial_{3} u_{2}\right]=-f-\operatorname{div}\left[\underset{\sim}{a}(\underset{\sim}{\hat{x}}) \underset{\sim}{\nabla} u_{0}\right], \\
\left.\partial_{3}\left[a_{33}(\underset{\sim}{\hat{x}}) \partial_{3} u_{2 k}\right]=-\operatorname{div}[\underset{\sim}{a} \underset{\sim}{\hat{x}}) \underset{\sim}{\nabla} u_{2 k-2}\right] \quad \text { for all } k \geq 2 .
\end{gathered}
$$


The boundary conditions on $\partial P_{ \pm}$are

$$
a_{33}(\underset{\sim}{\hat{x}}) \partial_{3} u_{0}=0, \quad a_{33}(\underset{\sim}{\hat{x}}) \partial_{3} u_{2}=\hat{x}_{3} g, \quad a_{33}(\underset{\sim}{\hat{x}}) \partial_{3} u_{2 k}=0 \text { for all } k \geq 2 .
$$

Equations (10)-(13) define a sequence of Neumann problems with respect to $\hat{x}_{3}$ in $(-1,1)$, parameterized by $\underset{\sim}{\hat{x}} \in \Omega$. Next we decompose

$$
u_{2 k}(\underline{\hat{x}})=\stackrel{\circ}{u}_{2 k}(\underline{\hat{x}})+\zeta_{2 k}\left(\stackrel{\hat{x}}{\sim}^{\prime} \quad \text { for all } k \in \mathbb{N},\right.
$$

where

$$
\int_{-1}^{1} \stackrel{\circ}{u}_{2 k}\left(\underset{\sim}{\hat{x}}, \hat{x}_{3}\right) d \hat{x}_{3}=0
$$

From the Dirichlet condition in (6), we would like to impose $u_{2 k}=0$ on $\partial P_{L}$, i.e.,

$$
\begin{array}{cc}
\zeta_{2 k}=0 & \text { on } \partial \Omega, \\
\stackrel{\circ}{u}_{2 k}=0 & \text { on } \partial P_{L} .
\end{array}
$$

However, this is not possible in general since only (15) can be imposed. Thus (16) does not hold in general, making necessary the introduction of correctors.

Note now that $\zeta_{2 k}, \stackrel{\circ}{u}_{2 k}$, and thus $u_{2 k}$, are uniquely determined from (10)-(15). In fact, from (10) and (13), we gather that $\stackrel{\circ}{0}_{0}=0$. To impose compatibility condition on (11) and (13), then

$$
\int_{-1}^{1} \operatorname{div}\left[\underset{\sim}{a}(\underset{\sim}{\hat{x}}) \underset{\sim}{\nabla} \zeta_{0}(\underset{\sim}{\hat{x}})\right] d \hat{x}_{3}=-\int_{-1}^{1} f\left(\underset{\sim}{\hat{x}}, \hat{x}_{3}\right) d \hat{x}_{3}-[g(\underset{\sim}{\hat{x}}, 1)+g(\underset{\sim}{\hat{x}},-1)],
$$

and from (15),

$$
\begin{gathered}
-2 \operatorname{div}\left[\underset{\sim}{a}(\underset{\sim}{\hat{x}}) \underset{\sim}{\nabla} \zeta_{0}(\underset{\sim}{\hat{x}})\right]=-\int_{-1}^{1} f\left(\underset{\sim}{\hat{x}}, \hat{x}_{3}\right) d \hat{x}_{3}-[g(\underset{\sim}{\hat{x}}, 1)+g(\underset{\sim}{\hat{x}},-1)] \text { in } \Omega, \\
\zeta_{0}=0 \text { on } \partial \Omega .
\end{gathered}
$$

Since $\stackrel{\circ}{u}_{0}=0$, then $u_{0}=\zeta_{0}$.

In general, from (12) and (13) with $k \geq 2$,

$$
\begin{gathered}
\left.-\operatorname{div}[\underset{\sim}{a} \underset{\sim}{\hat{x}}) \zeta_{2 k-2}(\underset{\sim}{x})\right]=0 \quad \text { in } \Omega, \\
\zeta_{2 k-2}=0 \quad \text { on } \partial \Omega,
\end{gathered}
$$

thus $\zeta_{2 k-2}=0$ for all $k \geq 2$.

From (12), (13),

$$
u_{0}=\zeta_{0}, \quad u_{2}=\stackrel{\circ}{u}_{2} \neq 0, \quad u_{4}=\stackrel{\circ}{u}_{4} \text {, etc. }
$$


Thus

$$
u^{\delta} \sim \zeta_{0}+\delta^{2} \stackrel{\circ}{u}_{2}+\delta^{4} \stackrel{\circ}{u}_{4}+\cdots
$$

Note that the first term in the asymptotic expansion (18) matches $w_{0}$, solution of (4).

Since $\stackrel{\circ}{u}_{2 k}$ does not vanish on $\partial P_{L}$, we introduce the boundary corrector

$$
U \sim \delta^{2} U_{2}+\delta^{4} U_{4}+\cdots
$$

where $U_{2 k} \in H^{1}(P)$ solves

$$
\begin{gathered}
-\delta^{2} \operatorname{div}\left[\underset{\sim}{a}(\underset{\sim}{\hat{x}}) \underset{\sim}{\nabla} U_{2 k}\right]-a_{33}(\underset{\sim}{\hat{x}}) \partial_{33} U_{2 k}=0 \quad \text { in } P, \\
\frac{\partial U_{2 k}}{\partial n}=0 \quad \text { on } \partial P_{ \pm}, \quad U_{2 k}=\stackrel{\circ}{u}_{2 k} \quad \text { on } \partial P_{L} .
\end{gathered}
$$

We finally conclude that the asymptotic expansion for $u(\delta)$ in $P$ is

$$
u(\delta) \sim \zeta_{0}+\delta^{2} \stackrel{\circ}{u}_{2}-\delta^{2} U_{2}+\delta^{4} \stackrel{\circ}{u}_{4}-\delta^{4} U_{4}+\cdots .
$$

Next we show some results that are necessary to estimate the modeling error. The result below follows from classical estimates. The constants are generally denoted by $c$, even if they are not the same in different occurrences. These constants are independent of $\delta$ but might depend on $\underset{\approx}{a}, a_{33}, \Omega$ and also on Sobolev norms of $f$ and $g$. We also assume that $\underset{\approx}{a}, a_{33}$ are smooth. Of course such hypothesis are not appropriate in practical applications, but they allow for an explicity rate of convergence. See the remark after Theorem 9 for a discussion on how one can proceed in the nonsmooth case.

The following classical regularity estimates follow.

Lemma 1. Let $\zeta_{0}$ and $\stackrel{\circ}{u}_{2 k}$ be defined as above, for $k \in \mathbb{N}$. Then there exists a constant $c$ such that

$$
\left\|\zeta_{0}\right\|_{H^{1}(\Omega)}+\left\|\stackrel{\circ}{u}_{2 k}\right\|_{H^{1}(P)} \leq c
$$

To estimate (19), we consider now the problem of finding $\Psi \in H^{1}(P)$ such that

$$
\begin{gathered}
\left.-\delta^{2} \operatorname{div}[\underset{\sim}{a} \underset{\sim}{\hat{x}}) \underset{\sim}{\nabla} \Psi\right]-a_{33}(\underset{\sim}{\hat{x}}) \partial_{33} \Psi=0 \quad \text { in } P, \\
\frac{\partial \Psi}{\partial n}=0 \quad \text { on } \partial P_{ \pm}, \quad \Psi=w \quad \text { on } \partial P_{L} .
\end{gathered}
$$

Lemma 2. Let $\Psi$ as in (21). Assume also that $\int_{-1}^{1} w\left(\underset{\sim}{\hat{x}}, \hat{x}_{3}\right) d \hat{x}_{3}=0$ on $\partial P_{L}$. Then there exists a constant $c$ such that

$$
\|\Psi\|_{L^{2}(P)} \leq c\left\|\partial_{3} \Psi\right\|_{L^{2}(P)}
$$


Proof. From the Neumann conditions on $\partial P_{ \pm}$, it follows that $\int_{-1}^{1} \partial_{33} \Psi d \hat{x}_{3}=0$ in $\Omega$. Thus, integrating the first equation in $(21)$ with respect to $\hat{x}_{3}$, we gather that

$$
\left.-\delta^{2} \int_{-1}^{1} \operatorname{div}[\underset{\sim}{a} \underset{\sim}{\hat{x}}) \underset{\sim}{\nabla} \Psi\right] d \hat{x}_{3}=0 \quad \text { in } \Omega
$$

and then

$$
-\delta^{2} \operatorname{div}[\underset{\sim}{a} \underset{\sim}{\hat{x}} \underset{\sim}{\nabla} \bar{\Psi}]=0 \quad \text { in } \Omega, \quad \bar{\Psi}=0 \quad \text { on } \partial \Omega
$$

where

$$
\bar{\Psi}(\underset{\sim}{\hat{x}})=\int_{-1}^{1} \Psi\left(\underset{\sim}{\hat{x}}, \hat{x}_{3}\right) d \hat{x}_{3} .
$$

Thus $\bar{\Psi} \equiv 0$ in $\Omega$. Then, from Poincaré's inequality, $\|\Psi\|_{L^{2}(P)} \leq c\left\|\partial_{3} \Psi\right\|_{L^{2}(\Omega)}$.

Lemma 3. Let $\Psi$ be the solution of (21), where $w \in W^{1, \infty}(P)$. Then there exists a constant $c$ such that

$$
\|\underline{\nabla} \Psi\|_{L^{2}(P)} \leq c \delta^{-1 / 2}\|w\|_{W^{1, \infty}(P)} .
$$

Proof. As in [18], let $\chi \in \mathcal{C}^{\infty}(\Omega)$ such that

$$
\chi(\underset{\sim}{\hat{x}})= \begin{cases}1 & \text { if } \operatorname{dist}(\underset{\sim}{\hat{x}}, \partial \Omega)<\delta, \\ 0 & \text { if } \operatorname{dist}(\underset{\sim}{\hat{x}}, \partial \Omega)>2 \delta,\end{cases}
$$

and

$$
\|\chi\|_{L^{2}(P)}^{2} \leq c \delta, \quad\|\underset{\sim}{\nabla} \chi\|_{L^{2}(P)}^{2} \leq \frac{c}{\delta}
$$

Let

$$
V_{w}(P):=\left\{v \in H^{1}(P):\left.v\right|_{\partial P_{L}}=w\right\}
$$

Since $\Psi-\chi w \in V(P)$,

$$
\delta^{2} \int_{P} \underset{\sim}{a}(\underset{\sim}{\hat{x}}) \underset{\sim}{\nabla} \Psi \underset{\sim}{\nabla}(\Psi-\chi w) d \underline{\hat{x}}+\int_{P} a_{33}(\underset{\sim}{\hat{x}}) \partial_{3} \Psi \partial_{3}(\Psi-\chi w) d \underline{\hat{x}}=0 .
$$

Thus, using (22),

$$
\begin{aligned}
& \delta^{2}\|\underset{\sim}{\nabla} \Psi\|_{L^{2}(P)}^{2}+\left\|\partial_{3} \Psi\right\|_{L^{2}(P)}^{2} \\
& \leq c \delta^{2}\|w \underset{\sim}{\nabla} \Psi\|_{L^{2}(P)}\|\underset{\sim}{\nabla} \chi\|_{L^{2}(p)}+\delta^{2}\|\underset{\sim}{\nabla} \Psi \underset{\sim}{\nabla} w\|_{L^{2}(P)}\|\chi\|_{L^{2}(P)}+\left\|\partial_{3} \Psi \partial_{3} w\right\|_{L^{2}(P)}\|\chi\|_{L^{2}(P)} \\
& \leq c \delta^{3 / 2}\|w\|_{W^{1, \infty}(P)}\|\underset{\sim}{\nabla} \Psi\|_{L^{2}(P)}+c \delta^{1 / 2}\left\|\partial_{3} w\right\|_{L^{\infty}(P)}\left\|\partial_{3} \Psi\right\|_{L^{2}(P)} \\
& \quad \leq c \delta^{1 / 2}\left(\delta^{2}\|\underset{\sim}{\nabla} \Psi\|_{L^{2}(P)}^{2}+\left\|\partial_{3} \Psi\right\|_{L^{2}(P)}^{2}\right)^{1 / 2}\|w\|_{W^{1, \infty}(P)} .
\end{aligned}
$$

Thus, $\|\underline{\nabla} \Psi\|_{L^{2}(P)} \leq c \delta^{-1 / 2}\|w\|_{W^{1, \infty}}$.

We need another technical result before proceeding with our estimates. 
Lemma 4. Let $F \in L^{2}(P)$ and $\Theta \in H^{1}(P)$ weak solution of

$$
\begin{gathered}
\left.-\delta^{2} \operatorname{div}[\underset{\sim}{a} \underset{\sim}{\hat{x}}) \underset{\sim}{\nabla} \Theta\right]-a_{33}(\underset{\sim}{\hat{x}}) \partial_{33} \Theta=F \quad \text { in } P, \\
\frac{\partial \Theta}{\partial n}=0 \quad \text { on } \partial P_{ \pm}, \quad \Theta=0 \quad \text { on } \partial P_{L} .
\end{gathered}
$$

Then there exists a constant $c$ such that

$$
\|\Theta\|_{H^{1}(P)} \leq c \delta^{-2}\|F\|_{L^{2}(P)} .
$$

Proof. Note that $\Theta \in V(P)$ is such that

$$
\delta^{2} \int_{P}[\underset{\sim}{a}(\underset{\sim}{\hat{x}}) \underset{\sim}{\nabla} \Theta] \cdot \underset{\sim}{\nabla} v d \underline{\hat{x}}+\int_{P} a_{33}(\underset{\sim}{\hat{x}}) \partial_{3} \Theta \partial_{3} v d \underline{\hat{x}}=\int_{P} F v d \underline{\hat{x}} \quad \text { for all } v \in V(P) .
$$

Making $v=\Theta$, from (2) and the Cauchy-Schwartz inequality we have that

$$
\delta^{2}\|\underset{\sim}{\nabla} \Theta\|_{L^{2}(P)}^{2}+\left\|\partial_{3} \Theta\right\|_{L^{2}(P)}^{2} \leq c\|F\|_{L^{2}(P)}\|\Theta\|_{L^{2}(P)} .
$$

Since $\left.\Theta\right|_{\partial P_{L}}=0$, the Poincaré's inequality holds and $\|\underline{\nabla} \Theta\|_{L^{2}(P)} \leq c \delta^{-2}\|F\|_{L^{2}(P)}$. The result follows from another application of the Poincaré's inequality.

Using Lemmas 2 and 3, we obtain an estimate for the solutions of (19).

Corollary 5. Assume that $U_{2 k}, k \in \mathbb{N}$, solve (19). Then

$$
\left\|U_{2 k}\right\|_{H^{1}(P)} \leq c \delta^{-1 / 2}
$$

We next estimate the residue $r=u^{\delta}-\left(\zeta_{0}+\delta^{2} \stackrel{\circ}{u}_{2}\right)$. We first note that

$$
\begin{gathered}
\left.-\delta^{2} \operatorname{div}[\underset{\sim}{a} \underset{\sim}{\hat{x}}) \underset{\sim}{\nabla} r\right]-a_{33}(\underset{\sim}{\hat{x}}) \partial_{33} r=\delta^{4} a_{33}(\underset{\sim}{\hat{x}}) \partial_{33} \stackrel{\circ}{4}_{4} \quad \text { in } P, \\
\frac{\partial r}{\partial n}=0 \quad \text { on } \partial P_{ \pm}, \quad r=-\delta^{2} \stackrel{\circ}{u}_{2} \quad \text { on } \partial P_{L} .
\end{gathered}
$$

The following result holds.

Theorem 6. Let $r$ as above. Then there exists a constant $c$ such that

$$
\|r\|_{H^{1}(P)} \leq c \delta^{3 / 2}
$$

Proof. From Lemmas 4, 2, and 3, it follows that

$$
\left.\|r\|_{H^{1}(P)} \leq c\left(\delta^{-1 / 2}\left\|\delta^{2} \stackrel{\circ}{u}_{2}\right\|_{W^{1, \infty}(P)}+\delta^{-2} \| \delta^{4} a \underset{\sim}{\hat{x}}\right) \partial_{33} \stackrel{\circ}{u}_{4} \|_{L^{2}(P)}\right) \leq c \delta^{3 / 2},
$$

and then the result holds. 
The following result presents an estimate for the difference between $u^{\delta}$ and the first term of the asymptotic expansion.

Corollary 7. Let $u^{\delta}$ be the solution of (1), and $\zeta_{0}$ be the solution of (17). Then

$$
\left\|u^{\delta}-\zeta_{0}\right\|_{H^{1}(P)} \leq c \delta^{3 / 2}
$$

Proof. Adding and subtracting $\delta^{2} \stackrel{\circ}{ }^{2}$, and using (20) and (23), we gather that

$$
\left\|u^{\delta}-\zeta_{0}\right\|_{H^{1}(P)} \leq\left\|u^{\delta}-\left(\zeta_{0}+\delta^{2} \stackrel{\circ}{u}_{2}\right)\right\|_{H^{1}(P)}+\delta^{2}\left\|\stackrel{\circ}{u}_{2}\right\|_{H^{1}(P)} \leq c \delta^{3 / 2}+c \delta^{2} .
$$

To estimate the modeling error, we need the following result [1].

Lemma 8. Let $w_{1} \in H_{0}^{1}(\Omega)$ be the solution of (5). Then

$$
\left\|w_{1}\right\|_{H^{1}(\Omega)} \leq c \delta^{1 / 2}
$$

Remark. It is also possible to show the above result by modifying the proofs of Lemmas 4 and 3 .

From Lemma 8, we have that $\left\|\delta \hat{x}_{3} w_{1}\right\|_{H^{1}(P)} \leq c \delta^{3 / 2}$. Let

$$
\tilde{u}(\delta)(\underline{\hat{x}})=\tilde{u}^{\delta}(\underline{x})=\zeta_{0}(\underset{\sim}{\hat{x}})+\delta \hat{x}_{3} w_{1}(\underset{\sim}{\hat{x}}) .
$$

Thus

$$
\|\tilde{u}(\delta)-u(\delta)\|_{H^{1}(P)} \leq\left\|\tilde{u}(\delta)-\zeta_{0}\right\|_{H^{1}(P)}+\left\|u(\delta)-\zeta_{0}\right\|_{H^{1}(P)} \leq c \delta^{3 / 2}+c \delta^{3 / 2} \leq c \delta^{3 / 2} .
$$

Thus, we can finally conclude the convergence of our continuous plate model with respect to the plate thickness.

Theorem 9. Let $u(\delta)$ be the solution for $(6)$ and $\tilde{u}(\delta)$ the model solution. Then there exists an $\delta$-independent constant such that

$$
\|\tilde{u}(\delta)-u(\delta)\|_{H^{1}(P)} \leq c \delta^{3 / 2} .
$$

Remark. Although the estimate of Theorem 9 requires the coefficients to be unduly smooth, a convergence result follows from [11, Theorem 1] (see also [5, Proposition A.1]), and assuming (2) is sufficient then. However, using such result, the rate of convergence with respect to $\delta$ does not come out naturally. 


\section{Multiscale Numerical SChemes}

The plate problem we consider here has rough coefficients, and the reduced model inherits such characteristic. Thus, although the PDEs in (4), (5) are much easier to solve than their original three-dimensional counterparts, they still pose a tough computational challenge. In fact, for highly heterogeneous materials, the coefficients $\underset{\sim}{a}(\cdot)$ and $a_{33}(\cdot)$ can be oscillatory, making the traditional finite element and difference methods almost useless. In (5) a new difficulty arises since the PDE is singularly perturbed with respect to $\delta$.

To overcome such troubles we employ two finite element methods of multiscale type. We first briefly describe the Residual Free Bubbles (RFB) method [7,8,16,22], which consists in enriching the usual finite element space of polynomials with bubbles, functions that vanish on the border of each element.

Consider the second order elliptic problem

$$
\mathcal{L} u=f \quad \text { in } \Omega, \quad u=0 \quad \text { on } \partial \Omega,
$$

where the differential operator $\mathcal{L}$ is defined by one of the equations below:

$$
\left.\mathcal{L} v=-\operatorname{div}[\underset{\sim}{a}(\underset{\sim}{x}) \underset{\sim}{\nabla} v], \quad \mathcal{L} v=-\frac{2 \delta^{3}}{3} \operatorname{div}[\underset{\sim}{a} \underset{\sim}{x}) \underset{\sim}{\nabla} v\right]+2 \delta a_{33}(\underset{\sim}{x}) v
$$

cf. (4), (5). Let $a: H_{0}^{1}(\Omega) \times H_{0}^{1}(\Omega) \rightarrow \mathbb{R}$ be the bilinear form associated with (24).

Let $\mathcal{T}_{h}$ be a regular partition of $\Omega$ into finite elements $K$. Associated with such partition, let $V_{1} \subset H_{0}^{1}(\Omega)$ be the space of continuous piecewise linear functions, and the bubble space

$$
V_{B}=\left\{v \in H_{0}^{1}(\Omega):\left.v\right|_{\partial K}=0 \text { for all } K \in \mathcal{T}_{h}\right\}
$$

The residual free bubble method consists in applying the Galerkin method in $V_{1} \oplus V_{B}$, i.e, we search for $u_{1}+u_{b}$, where $u_{1} \in V_{1}, u_{b} \in V_{B}$, and

$$
a\left(u_{1}+u_{b}, v_{1}+v_{b}\right)=\left(f, v_{1}+v_{b}\right), \quad \text { for all } v_{1}+v_{b} \in V_{1} \oplus V_{B} .
$$

The basic idea now is to apply a static condensation trick and write $u_{b}$ in terms of $u_{1}$. Testing (25) with functions in $V_{1}$ only, we gather that $u_{b}=\mathcal{L}_{*}^{-1} f-\mathcal{L}_{*}^{-1} \mathcal{L} u_{1}$, where $\mathcal{L}_{*}^{-1}$ : $L^{2}(\Omega) \rightarrow V_{B}$ is such that if $v=\mathcal{L}_{*}^{-1} g$, for $g \in L^{2}(\Omega)$, then

$$
\mathcal{L} v=g \quad \text { in } K, \quad v=0 \quad \text { on } \partial K
$$

for all $K \in \mathcal{T}_{h}$. Thus

$$
a\left(u_{1}-\mathcal{L}_{*}^{-1} \mathcal{L} u_{1}, v_{1}\right)=\left(f, v_{1}\right)-a\left(\mathcal{L}_{*}^{-1} f, v_{1}\right), \quad \text { for all } v_{1} \in V_{1} .
$$


In terms of finite element implementation, if $\left\{\psi_{i}\right\}_{i=1}^{N}$ is a basis of $V_{1}$, where $\psi_{i}$ are the usual piecewise linear functions, we define $\lambda_{i}$ such that

$$
\mathcal{L} \lambda_{i}=0 \quad \text { in } K, \quad \lambda_{i}=\psi_{i} \quad \text { on } \partial K .
$$

Next, if $u_{1}=\sum_{i=1}^{N} u_{i} \psi_{i}$, then

$$
\sum_{i=1}^{N} a\left(\lambda_{i}, \psi_{j}\right) u_{i}=\left(f, \psi_{j}\right)-a\left(\mathcal{L}_{*}^{-1} f, \psi_{j}\right), \quad j=1, \ldots, N .
$$

The other method that we used to discretize our model is the Multiscale Finite Element Method (MsFEM) [18-20]. In its present form, the MsFEM consists in using the Galerkin method with the subspace generated by functions $\left\{\lambda_{i}\right\}_{i=1}^{N}$. Actually, both methods are closely related [22], and yield similar numerical results.

Remark. Regarding the oscillatory reaction-diffusion problem (5), an alternative would be to proceed as in [17], and propose alternative boundary conditions for the multiscale base functions.

Remark. The computational cost of finding the basis functions $\lambda_{i}$ is quite a drawback of both RFB and MsFEM. Nevertheless, these methods are still cheaper than solving the original PDE via traditional numerical schemes [20, Section 4.2], specially since the local problems can be solved in parallel. Moreover, as reported in [20], the overall solution is rather insensitive to the resolution of the basis functions. Of course, it is also possible to exploit eventual periodicities of the coefficients and significantly reduce the amount of computation. For instance, in the tests considered in Section 5, only one local problem had to be solved.

\section{NUMERICAL TESTS}

In the present section, we show some numerical results related to the problems (4), (5). In particular, using multiscale schemes for the double parameter problem (4), we show computational results that we believe are new in the literature.

We assume in $(4),(5)$ that $\Omega=(0,1) \times(0,1)$, and $\underset{\approx}{a}(\cdot)=a(\cdot) \underset{\approx}{I}$, where $\underset{\approx}{I}$ is the $2 \times 2$ identity matrix, and that $a_{33}(\cdot)=a(\cdot)$ are periodic with respect to $x$ and $y$ with periodicity $\varepsilon$. In all problems below we use a subgrid of $128 \times 128$ elements, and always choose the coarse mesh size as a multiple of $\varepsilon$, allowing considerable computational savings, as pointed out in the last remark of Section 4. Furthermore, to check the accuracy of the methods we computed "exact" solutions using overrefined meshes, since we do not have analytical expressions for them. 


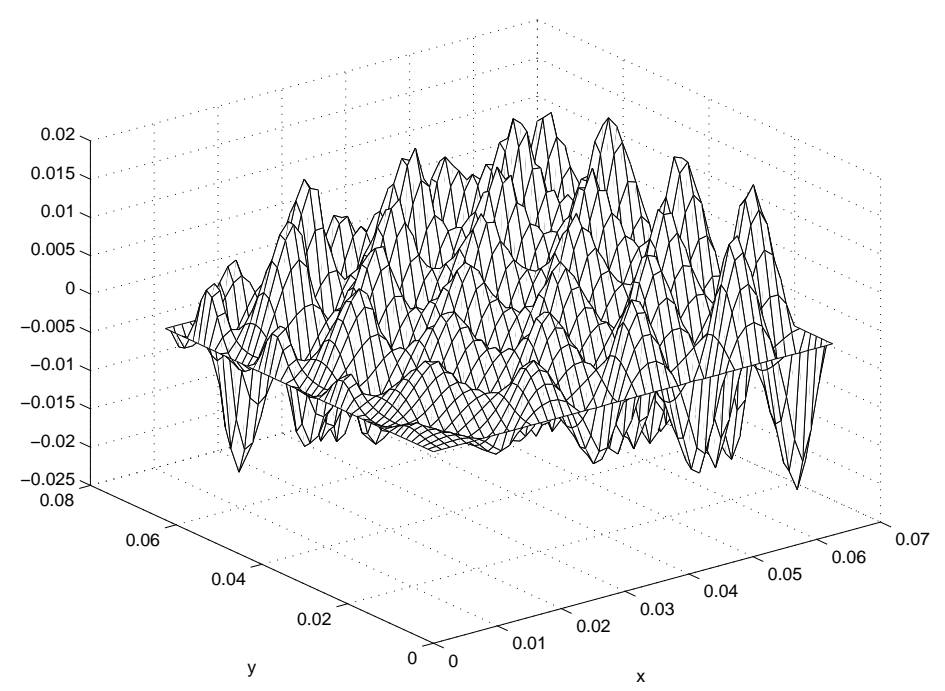

Figure 1. Plot for $\psi-\lambda$.

We use both the RFB and MsFEM to approximate (4). We assume

$$
\begin{gathered}
a\left(x_{1}, x_{2}\right)=\frac{9}{2} \sin \left(2 \pi \epsilon^{-1} x_{1}\right) \cos \left(2 \pi \epsilon^{-1} x_{2}\right)+\frac{11}{2}, \quad f^{\delta}(\underset{\sim}{x})=0, \\
g^{\delta}(\underset{\sim}{x}, \delta)+g^{\delta}(\underset{\sim}{x},-\delta)=2 \delta,
\end{gathered}
$$

with $\varepsilon=1 / 32$. Note that with such choices for $f^{\delta}$ and $g^{\delta}$ the solution of (4) does not depend on the value of $\delta$.

We start by showing how far the multiscale basis function departs from the traditional linear function, plotting $\psi-\lambda$ in Figure 1 for a fixed element $K=[0,1 / 16] \times[0,1 / 16]$. Then, Figure 2 shows $\lambda$, and Figure 3 shows its level curves. Next, Figure 4 displays the profile at $x=y$ of various approximate solutions and also an "exact" solution, obtained with the aid of an overrefined mesh. It is possible to see then that the finite element method with piecewise linear functions fails to deliver a good approximation.

We next consider $\epsilon=1 / 64$ and consider the convergence of the methods in the range $\varepsilon \leq h$. Let

$$
\begin{gathered}
a\left(x_{1}, x_{2}\right)=\frac{2+p \sin \left(2 \pi \epsilon^{-1} x_{1}\right)}{2+p \cos \left(2 \pi \epsilon^{-1} x_{2}\right)}+\frac{2+\sin \left(2 \pi \epsilon^{-1} x_{2}\right)}{2+p \sin \left(2 \pi \epsilon^{-1} x_{1}\right)}, \quad f^{\delta}(\underset{\sim}{x})=0, \\
g^{\delta}(\underset{\sim}{x}, \delta)+g^{\delta}(\underset{\sim}{x},-\delta)=-2 \delta,
\end{gathered}
$$

for $p=1.8$. We compare our the results in Figure 5 in the $l^{2}$ norm given by $\left\|u_{h}\right\|_{l^{2}(\Omega)}=$ $\left(\sum_{i=1}^{N} u_{h}\left(x_{\sim}\right)^{2} h^{2}\right)^{1 / 2}$. Such norm is equivalent to $\|\cdot\|_{L^{2}(\Omega)}$ in $V_{1}$ [6]. We again used a refined mesh to find a well resolved approximation to the exact solution. 


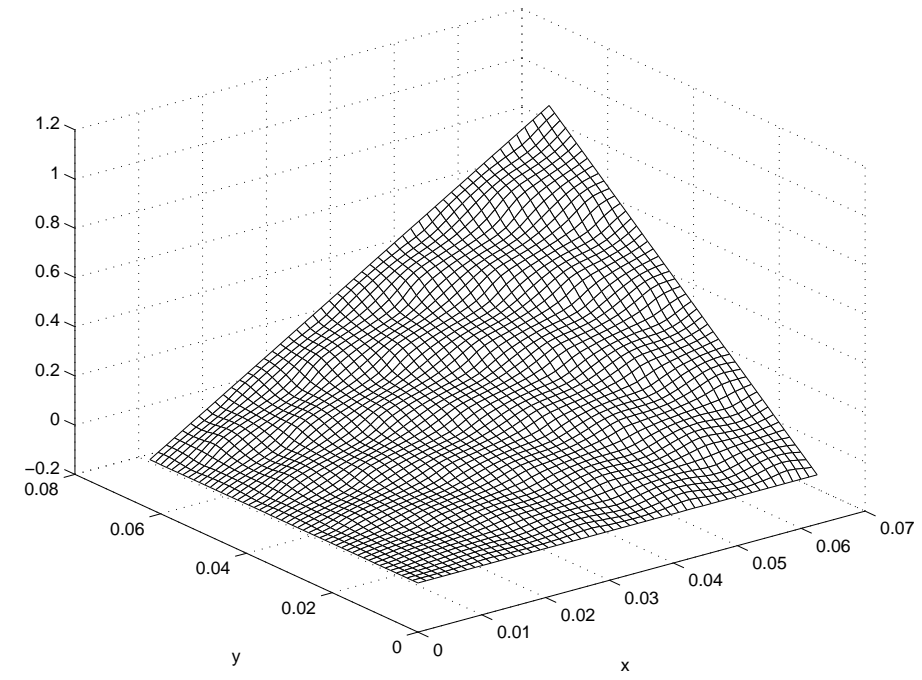

Figure 2. Basis function $\lambda$.

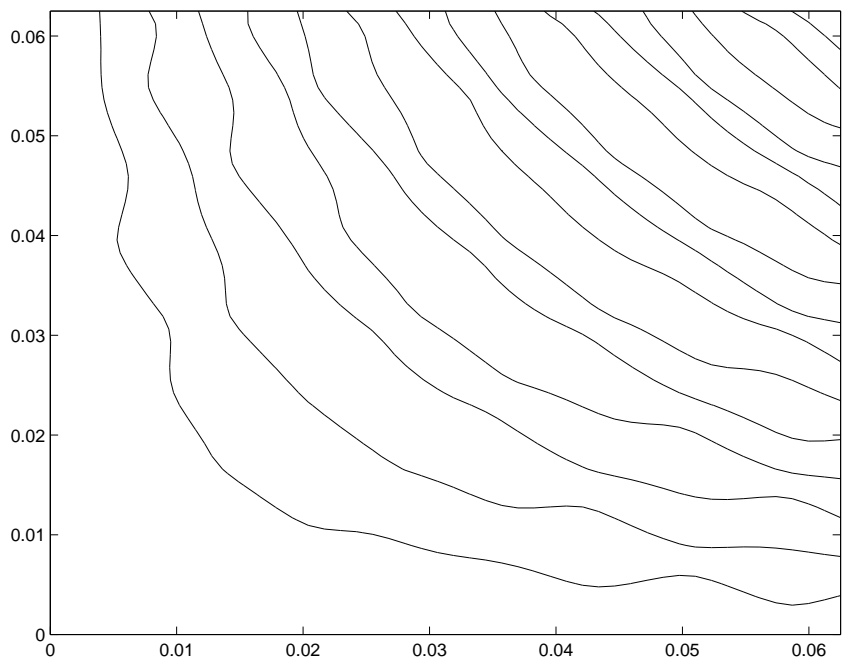

Figure 3. Level curves for $\lambda$.

Note that Galerkin with piecewise linear functions simply does not appear to converge if $\varepsilon \leq h$, while the multiscale methods perform quite well within such range. Of course, if $h \ll \varepsilon$, the convergence curves for the Galerkin method with piecewise linears recover its usual looks.

We next perform some numerical tests using the MsFEM in (5), again remarking that, to the best of our knowledge, such method was never tested in this problem. Let

$$
\begin{aligned}
a\left(x_{1}, x_{2}\right)=a_{33}\left(x_{1}, x_{2}\right)= & \frac{9}{2} \sin \left(2 \pi \epsilon^{-1} x_{1}\right) \cos \left(2 \pi \epsilon^{-1} x_{2}\right)+\frac{11}{2}, \quad f^{\delta}(\underset{\sim}{x})=0, \\
& g^{\delta}(\underset{\sim}{x},-\delta)-g^{\delta}(\underset{\sim}{x}, \delta)=2,
\end{aligned}
$$




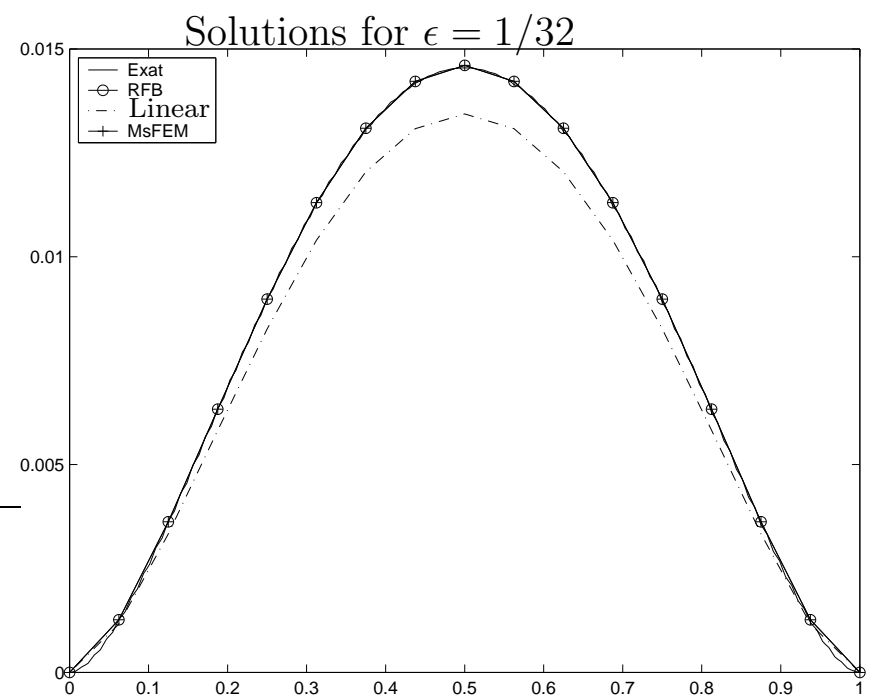

Figure 4. Diagonal profiles for exact, RFB, MsFEM and Linear FEM solutions.

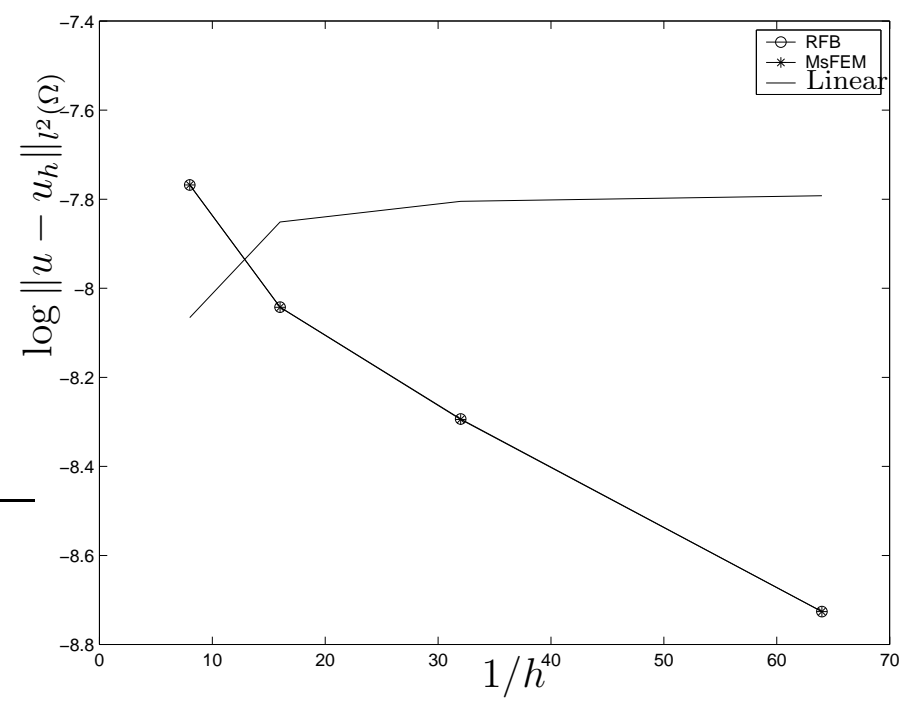

FiguRE 5. $\log \left\|u-u_{h}\right\|_{l^{2}(\Omega)}$ for $\epsilon=1 / 64$.

where $\epsilon=1 / 32$. For $\delta=10^{-1}$, we show $\psi-\lambda$ and $\lambda$ in Figures 6 and 7 , for the local problem in $K=[0,1 / 16] \times[0,1 / 16]$, with a rectangular submesh of $128 \times 128$ elements. In Figure 8 we show the level curves of $\lambda$. Observe the onset of strong boundary layers due to the singular perturbed flavor of the problem.

Finally, in Figure 9, we compare "exact" and approximate solutions for $\omega_{0}+\delta \omega_{1}$, when $\delta=$ $10^{-3}$. The "exact" solution was again obtained using a refined mesh. Again the performance of the MsFEM is good, while the traditional finite element method fails. 


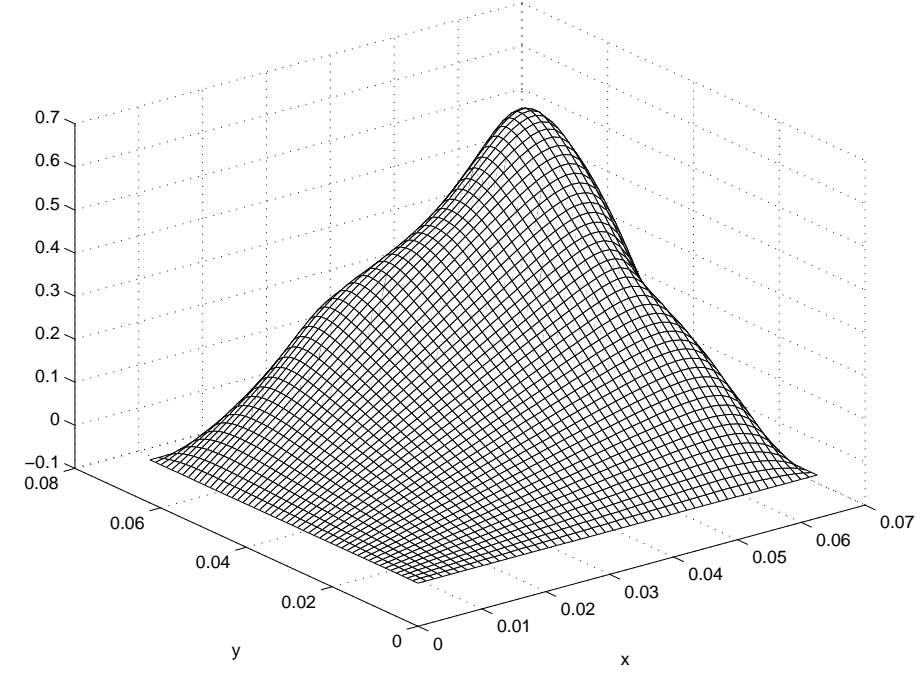

FiguRE 6. function $\psi-\lambda$.

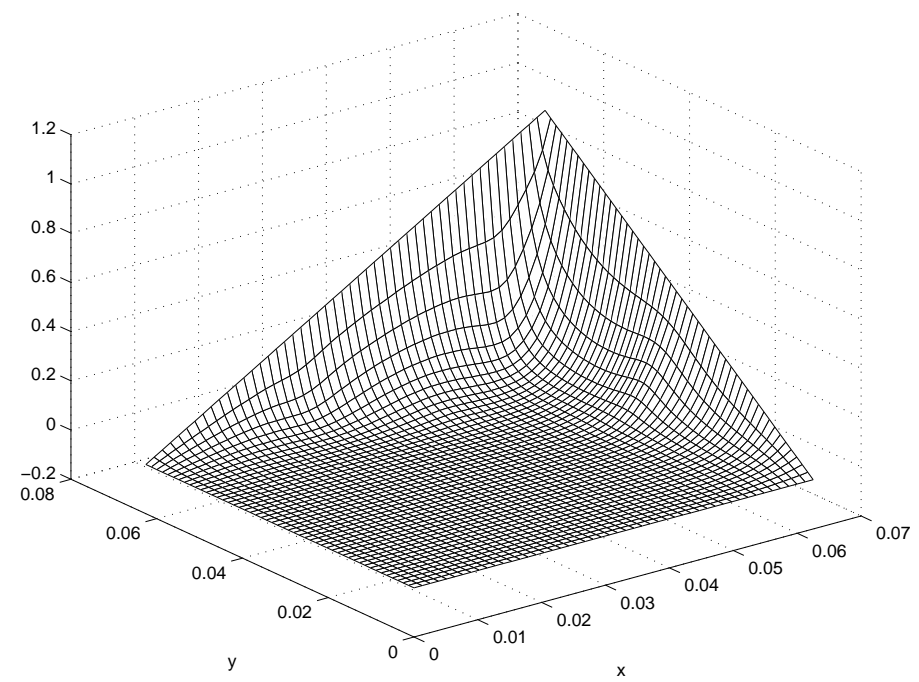

Figure 7. Basis function $\lambda$.

Remark. The numerical results above are not related to the convergence result in Theorem 9 . Although numerical examples substantiating the approximation properties of our model would be very interesting, that is certainly a daunting computational task. For the linearly elastic problem, but with homogeneous materials, such endeavor was considered in [15]. See also [14]. 


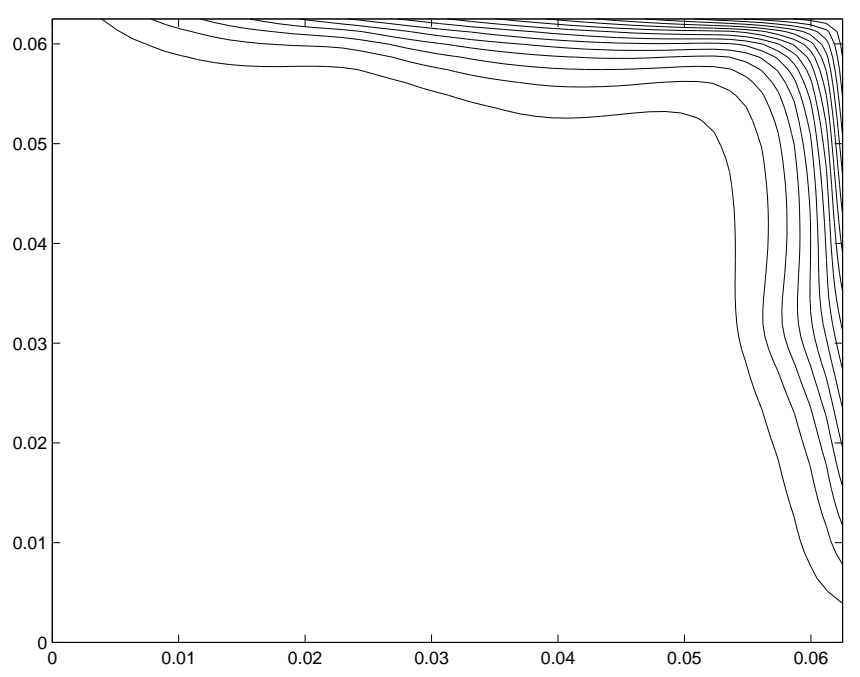

Figure 8. Level curves for $\lambda$.

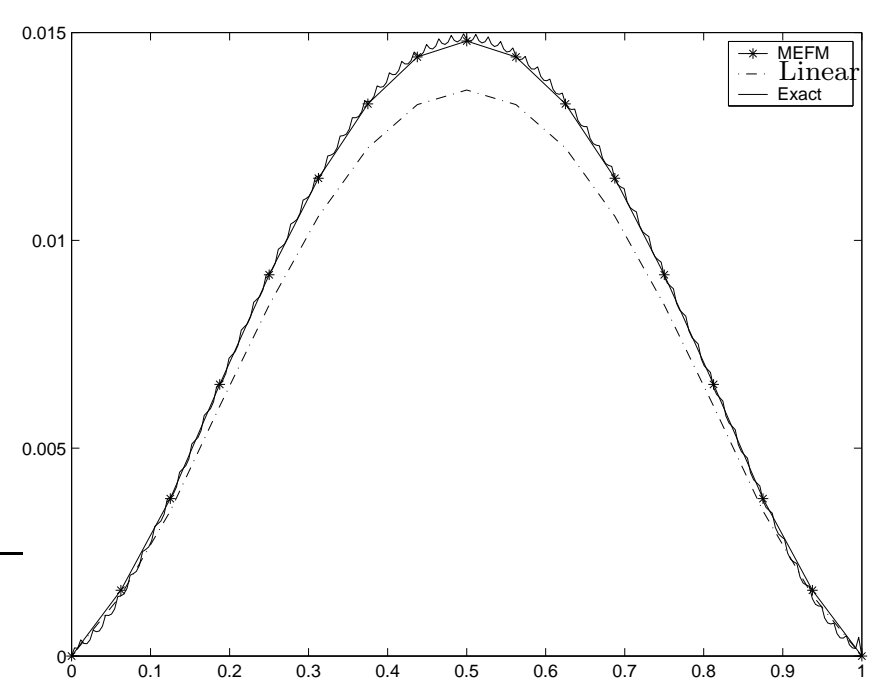

Figure 9. Diagonal profiles for exact, MsFEM and Linear FEM solutions.

\section{Appendix}

From its definition, $\left.\tilde{u}^{\delta} \underset{\sim}{x}, x_{3}\right)$ solves

$$
\int_{P^{\delta}} \underline{A}(\underset{\sim}{x}) \underline{\nabla} \tilde{u}^{\delta} \underline{\nabla} \tilde{v} d \underline{x}=\int_{P^{\delta}} f^{\delta} \tilde{v} d \underline{x}+\int_{\partial P_{ \pm}^{\delta}} g^{\delta} \tilde{v}, d \underline{x} \quad \text { for all } \tilde{v} \in V_{1}\left(P^{\delta}\right) .
$$


Using (3), where $w_{0}, w_{1} \in H_{0}^{1}(\Omega)$, and substituting $\tilde{v}\left(\underset{\sim}{x}, x_{3}\right)=v_{0}(\underset{\sim}{x})+v_{1}(\underset{\sim}{x}) x_{3} \in V_{1}\left(P^{\delta}\right)$, in $(27)$, we gather that

$$
\begin{gathered}
\left.\int_{P^{\delta}} \underset{\sim}{A} \underset{\sim}{x}\right) \underline{\nabla}\left[w_{0}(\underset{\sim}{x})+w_{1}(\underset{\sim}{x}) x_{3}\right] \cdot \underline{\nabla} v_{0}(\underset{\sim}{x}) d \underline{x}=\int_{P^{\delta}} f^{\delta}(\underline{x}) v_{0}(\underset{\sim}{x}) d \underline{x}+\int_{\partial P_{ \pm}^{\delta}} g^{\delta}(\underline{x}) v_{0}(\underset{\sim}{x}) d \underline{x}, \\
\left.\int_{P^{\delta}} \underset{\sim}{A} \underset{\sim}{x}\right) \underline{\nabla}\left[w_{0}(\underset{\sim}{x})+w_{1}(\underset{\sim}{x}) x_{3}\right] \cdot \underline{\nabla}\left[v_{1}(\underset{\sim}{x}) x_{3}\right] d \underline{x}=\int_{P^{\delta}} f^{\delta}(\underline{x}) v_{1}(\underset{\sim}{x}) x_{3} d \underline{x}+\int_{\partial P_{ \pm}^{\delta}} g^{\delta}(\underline{x}) v_{1}(\underset{\sim}{x}) x_{3} d \underline{x},
\end{gathered}
$$

for all $v_{0}, v_{1} \in H_{0}^{1}(\Omega)$. Integrating with respect to $x_{3}$,

$$
\begin{array}{r}
2 \delta \int_{\Omega} \underset{\sim}{a}(\underset{\sim}{x}) \underset{\sim}{\nabla} w_{0}(\underset{\sim}{x}) \underset{\sim}{\nabla} v_{0}(\underset{\sim}{x}) d \underset{\sim}{x}=\int_{\Omega} \int_{-\delta}^{\delta} f^{\delta}(\underline{x}) v_{0}(\underset{\sim}{x}) d x_{3} d \underset{\sim}{x}+\int_{\Omega}\left[g^{\delta}(\underset{\sim}{x}, \delta)+g^{\delta}(\underset{\sim}{x},-\delta)\right] v_{0}(\underset{\sim}{x}) d \underset{\sim}{x}, \\
\left.\frac{2 \delta^{3}}{3} \int_{\Omega} \underset{\sim}{a} \underset{\sim}{x}\right) \underset{\sim}{\nabla} w_{1} \underset{\sim}{\nabla} v_{1} d \underset{\sim}{x}+2 \delta \int_{\Omega} a_{33}(\underset{\sim}{x}) w_{1} v_{1} \underset{\sim}{d x}=\int_{\Omega} \int_{-\delta}^{\delta} f^{\delta}\left(\underset{\sim}{x}, x_{3}\right) x_{3} v_{1}(\underset{\sim}{x}) d x_{3} d \underset{\sim}{x} \\
+\delta \int_{\Omega}\left[g^{\delta}(\underset{\sim}{x}, \delta)-g^{\delta}(\underset{\sim}{x},-\delta)\right] v_{1}(\underset{\sim}{x}) d \underset{\sim}{x},
\end{array}
$$

and (4), (5) follow.

\section{REFERENCES}

[1] Stephen M. Alessandrini, Douglas N. Arnold, Richard S. Falk, and Alexandre L. Madureira, Derivation and justification of plate models by variational methods, Plates and shells (Québec, QC, 1996), CRM Proc. Lecture Notes, vol. 21, Amer. Math. Soc., Providence, RI, 1999, pp. 1-20. MR 1696513 (2000j:74055)

[2] Douglas N. Arnold and Alexandre L. Madureira, Asymptotic estimates of hierarchical modeling, Math. Models Methods Appl. Sci. 13 (2003), no. 9, 1325-1350. MR 2005646 (2004j:35074)

[3] Douglas N. Arnold, Alexandre L. Madureira, and Sheng Zhang, On the range of applicability of the Reissner-Mindlin and Kirchhoff-Love plate bending models, J. Elasticity 67 (2002), no. 3, 171-185 (2003). MR 1997951 (2004e:74053)

[4] Ferdinando Auricchio, Carlo Lovadina, and Alexandre L. Madureira, An asymptotically optimal model for isotropic heterogeneous linearly elastic plates, M2AN Math. Model. Numer. Anal. 38 (2004), no. 5, 877-897. MR 2104433 (2005i:74051)

[5] F. Auricchio, P. Bisegna, and C. Lovadina, Finite element approximation of piezoelectric plates, Internat. J. Numer. Methods Engrg. 50 (2001), no. 6, 1469-1499. MR 1811534 (2001m:74063)

[6] Dietrich Braess, Finite elements, Cambridge University Press, Cambridge, 1997. Theory, fast solvers, and applications in solid mechanics; Translated from the 1992 German original by Larry L. Schumaker. MR 1463151 (98f:65002)

[7] Franco Brezzi and Alessandro Russo, Choosing bubbles for advection-diffusion problems, Math. Models Methods Appl. Sci. 4 (1994), no. 4, 571-587. MR 1291139 (95h:76079)

[8] F. Brezzi, L. P. Franca, T. J. R. Hughes, and A. Russo, $b=\int g$, Comput. Methods Appl. Mech. Engrg. 145 (1997), no. 3-4, 329-339. MR 1456019 (98g:65086) 
[9] D. Caillerie, Homogénéisation des équations de la diffusion stationnaire dans les domaines cylindriques aplatis, RAIRO Anal. Numér. 15 (1981), no. 4, 295-319 (French, with English summary). MR 642495 (83g:80003)

[10] _ Thin elastic and periodic plates, Math. Methods Appl. Sci. 6 (1984), no. 2, 159-191. MR 751739 (86c:73020)

[11] D. Chenais and J.-C. Paumier, On the locking phenomenon for a class of elliptic problems, Numer. Math. 67 (1994), no. 4, 427-440. MR 1274440 (95g:65147)

[12] Philippe G. Ciarlet, Mathematical elasticity. Vol. II, Studies in Mathematics and its Applications, vol. 27, North-Holland Publishing Co., Amsterdam, 1997. Theory of plates. MR 1477663 (99e:73001)

[13] Doina Cioranescu and Jeannine Saint Jean Paulin, Homogenization of reticulated structures, Applied Mathematical Sciences, vol. 136, Springer-Verlag, New York, 1999. MR 1676922 (2000d:74064)

[14] Monique Dauge, Erwan Faou, and Zohar Yosibash, Plates and Shells: Asymptotic expansions and hierarchical models (René de Borst and Thomas Hughes Erwin Stein, ed.), Encyclopedia of Computational Mechanics, John Wiley\&Sons, 2004, pp. 83-92.

[15] Monique Dauge and Zohar Yosibash, Boundary layer realization in thin elastic three-dimensional domains and two-dimensional Hierarchic plate models, International Journal of Solids and Structures $\mathbf{3 7}$ (2000), no. 17, 2443-2471.

[16] Leopoldo P. Franca, An overview of the residual-free-bubbles method, Numerical methods in mechanics (Concepción, 1995), Pitman Res. Notes Math. Ser., vol. 371, Longman, Harlow, 1997, pp. 83-92. MR 1485802

[17] Leopoldo P. Franca, Alexandre L. Madureira, and Frederic Valentin, Towards multiscale functions: enriching finite element spaces with local but not bubble-like functions, Comput. Methods Appl. Mech. Engrg. 194 (2005), no. 27-29, 3006-3021. MR 2142535 (2006a:65159)

[18] Thomas Y. Hou, Numerical approximations to multiscale solutions in partial differential equations, Frontiers in numerical analysis (Durham, 2002), Universitext, Springer, Berlin, 2003, pp. 241-301. MR 2006969 (2004m:65219)

[19] Thomas Y. Hou, Xiao-Hui Wu, and Zhiqiang Cai, Convergence of a multiscale finite element method for elliptic problems with rapidly oscillating coefficients, Math. Comp. 68 (1999), no. 227, 913-943. MR 1642758 (99i:65126)

[20] Thomas Y. Hou and Xiao-Hui Wu, A multiscale finite element method for elliptic problems in composite materials and porous media, J. Comput. Phys. 134 (1997), no. 1, 169-189. MR 1455261 (98e:73132)

[21] Alexandre L. Madureira, Hierarchical modeling based on mixed principles: asymptotic error estimates, Math. Models Methods Appl. Sci. 15 (2005), no. 7, 985-1008. MR 2151796 (2006a:74057)

[22] Giancarlo Sangalli, Capturing small scales in elliptic problems using a residual-free bubbles finite element method, Multiscale Model. Simul. 1 (2003), no. 3, 485-503 (electronic). MR 2030161 (2004m:65202)

Departamento de Matemática Aplicada, Laboratório Nacional de ComputaÇão Científica, Av. Getúlio Vargas, 333, 25651-070 Petrópolis - RJ, Brazil

E-mail address: carol@lncc.br

E-mail address: alm@lncc.br 